\title{
Function and mechanism of microRNA-210 in acute cerebral infarction
}

\author{
JUN WANG ${ }^{1,2}$, YUEZHAN ZHANG ${ }^{1,3}$ and FENG XU ${ }^{1}$ \\ ${ }^{1}$ Department of Emergency, The First Affiliated Hospital of Soochow University, Suzhou, Jiangsu 215000; \\ ${ }^{2}$ Department of Emergency, Taizhou People's Hospital, Taizhou, Jiangsu 225300; ${ }^{3}$ Department of Emergency, \\ Lianyungang TCM Hospital Affiliated to Nanjing University of Chinese Medicine, Nanjing, Jiangsu 222000, P.R. China
}

Received April 22, 2017; Accepted October 20, 2017

DOI: $10.3892 / \mathrm{etm} .2017 .5577$

\begin{abstract}
Acute cerebral infarction (ACI) is a common cerebrovascular disease. Previous studies have indicated that microRNAs (miRs) are aberrantly expressed in patients with ACI. However, the functions of miRs in the pathogenesis of ACI still require further investigation. The aim of the present study was to investigate the function of miR-210 in ACI and its associated mechanism. The expression of miR-210 in the serum of 40 patients with ACI and 40 normal controls was examined using reverse transcription-quantitative polymerase chain reaction (RT-qPCR). Then, human umbilical vein endothelial cells (HUVECs) were treated with serum from patients with ACI or healthy volunteers, and a CCK-8 assay was performed to examine cell proliferation. Next, cells were stained with $\mathrm{PI} /$ Annexin $\mathrm{V}$, and the apoptosis rate was examined using flow cytometry. Furthermore, cells were harvested and lysed, and RT-qPCR and western blotting assays were performed to compare the expression of vascular endothelial growth factor (VEGF), Notch1 and Hes1 in different groups. It was observed that the expression of miR-210 was significantly increased in the serum of patients with ACI compared with normal controls $(\mathrm{P}<0.01)$, and receiver operating characteristic curve analysis indicated that the area under the curve for miR-210 was 0.799 (95\% confidence interval, 0.700-0.899), the optimum cut-off point was 1.397 , and the sensitivity and specificity at the cut-off point were 62.5 and $87.5 \%$, respectively. Furthermore, serum from patients with ACI induced a significant increase in proliferation $(\mathrm{P}<0.05$ at $48 \mathrm{~h}, \mathrm{P}<0.01$ at $72 \mathrm{~h})$ and a significant decrease in the apoptosis rate of HUVECs $(\mathrm{P}<0.01)$. In addition, serum from patients with ACI significantly increased the expression of VEGF, Notch1 and Hes1 at the mRNA and
\end{abstract}

Correspondence to: Dr Feng Xu, Department of Emergency, The First Affiliated Hospital of Soochow University, 188 Shizi Street, Suzhou, Jiangsu 215000, P.R. China

E-mail: dr_fengxu@163.com

Key words: micoRNA-210, acute cerebral infarction, human umbilical vein endothelial cells, proliferation, apoptosis protein level (all $\mathrm{P}<0.01$ with the exception of Notch1 mRNA expression, $\mathrm{P}>0.05$ ). In conclusion, these results demonstrate that miR-210 is upregulated in the serum of patients with ACI, and miR-210 may be involved in the pathogenesis of ACI through regulating the proliferation and apoptosis of endothelial cells.

\section{Introduction}

In recent years, the incidence of cerebrovascular disease has increased worldwide (1). Stroke is the second leading cause of mortality among people $>60$ years old worldwide. In China, the incidence of new cases of stroke is 2.5 million/year (2). Acute cerebral infarction (ACI) is cerebral infarction that occurs within 6 to $24 \mathrm{~h}$ and is caused by sudden occlusion of the cerebral artery, which can induce death of brain tissue (3). If rapid, efficient medical treatment is not administered, ACI can lead to severe sequelae and an increased rate of mortality among patients (4). Therefore, it is critical to identify ACI-specific serum biomarkers for the early diagnosis of ACI, even before magnetic resonance imaging (MRI) or computed tomography tests, to allow physicians to make rapid clinical decisions.

Previous studies have indicated that brain damage after ACI can be reflected by changes in certain serum biomarkers (including S100 protein, neuron specific enolase and cystatin C) (5-7). However, as proteins, these biomarkers consist of complicated components and degrade easily. Therefore, identifying novel, specific and stable serum biomarkers for the early diagnosis of ACI is now a key challenge for researchers and physicians in the field of cerebrovascular disease.

MicroRNAs (miRs) are a class of single-strand, endogenous, non-coding RNAs that are 22 nucleotides in length. In 1993, Lee et al (8) first discovered miRs in Caenorhabditis elegans. In recent years, miRs have been demonstrated to be involved in numerous biological processes, including cell proliferation, differentiation and apoptosis, and the incidence and progress of diseases (9-11). Previous studies have indicated that the expression of miRs in the serum is stable, and the method for detecting circulating miRs is sensitive and accurate. This implies that miRs have the potential to become novel biomarkers for the diagnosis of numerous diseases (12-14). In 
the case of ACI, aberrant expression of certain miRs in the brain tissue and peripheral blood of patients has also been observed, suggesting that miRs may be involved in the pathogenesis of ACI (15-17).

The aberrant expression of miR-210 has been identified in the blood of patients with ACI (18). However, the underlying mechanism of this requires further investigation. The present study aimed to elucidate the role of miR-210 in ACI and its associated mechanism. The serum expression of miR-210 was compared between patients with ACI and healthy controls, and the effects of miR-210 on proliferation and apoptosis of endothelial cells were investigated.

\section{Materials and methods}

Patients. In the present study, 40 patients were enrolled who had been diagnosed with ACI at the Department of Emergency, Taizhou People's Hospital (Taizhou, China), between January 2016 and October 2016. A total of 40 healthy volunteers were recruited from the Medical Examination Center of Taizhou People's Hospital and served as the controls. The clinical information of patients is presented in Table I. Venous blood samples $(\sim 5-8 \mathrm{ml})$ were drawn from all participants. The serum of patients and volunteers was isolated by centrifuging at $300 \mathrm{x} \mathrm{g}$ at $4^{\circ} \mathrm{C}$ for $20 \mathrm{~min}$, and then collected and stored at $-80^{\circ} \mathrm{C}$ until analysis. The present study was approved by the Ethics Committee of Taizhou People's Hospital, and all participants signed informed consent forms. The inclusion criteria were as follows: 1) Presence of ischemic lesion compatible with the pathological and imaging characteristics of the vasculature in the central nervous system (CNS) or the presence of clinical evidence for ischemic injury of the CNS; 2) presence of neurological deficits lasting more than $24 \mathrm{~h}$ due to ischemic lesions confirmed on conventional MRI of the brain. Patients were excluded if any other CNS disease was identified, based on medical history.

Cell culture. Human umbilical vein endothelial cells (HUVECs) were purchased from Bnbio (Beijing, China). Cells were cultured in RPMI-1640 (Thermo Fisher Scientific, Inc., Waltham, MA, USA) supplemented with $10 \%$ fetal bovine serum (FBS; Thermo Fisher Scientific, Inc.), $100 \mathrm{U}$ penicillin/ml and $100 \mathrm{mg}$ streptomycin $/ \mathrm{ml}$, in a humidified incubator at $37^{\circ} \mathrm{C}$ with $5 \% \mathrm{CO}_{2}$ until $70-80 \%$ confluence. The control group was treated with RPMI-1640 supplemented with $10 \%$ FBS, the patient group was treated with RPMI-1640 supplemented with $10 \%$ serum from patients, and the healthy volunteer group was treated with RPMI-1640 supplemented with $10 \%$ serum from the healthy volunteers in a humidified incubator at $37^{\circ} \mathrm{C}$ with $5 \% \mathrm{CO}_{2}$ for $48 \mathrm{~h}$.

Cell transfection. HUVECs $\left(2 \times 10^{5}\right)$ were seeded onto 6-well plates until they reached $60-70 \%$ confluence and transfected with $50 \mathrm{nmol}$ miR-210 mimics or miR-210 mimic negative controls (purchased from GenePharma Co., Ltd., Shanghai, China) using Lipofectamine 2000 (Thermo Fisher Scientific, Inc.), according to the manufacturer's protocol. The cells were harvested at $72 \mathrm{~h}$ after transfection. The sequences were as follows: miR-210 mimics sense 5'-CUGUGCGUGUGA CAGCGGCUGA-3' and miR-210 mimics antisense 5'-AGC
CGCUGUCACACGCACAGUU-3', miR-210 mimic negative controls (scrambled) sense 5'-UUCUCCGAACGUGUC ACGUTT-3' and miR-210 mimic negative controls antisense 5'-ACGUGACACGUUCGGAGAATT-3'.

Cell proliferation analysis. The proliferation rate of HUVECs with different treatments was determined using a CCK-8 proliferation assay kit (Sigma-Aldrich; Merck KGaA, Darmstadt, Germany), according to the manufacturer's protocol.

Cell apoptosis analysis. For the apoptosis analysis, HUVECs in different groups were stained with a PI/Annexin V-FITC apoptosis detection kit (BD Biosciences, San Jose, CA, USA) and analyzed on a BD FACSCalibur flow cytometer (BD Biosciences) according to the manufacturer's protocols. The results of the flow cytometry analysis were analyzed using FlowJo software (version 9.7; FlowJo LLC, Ashland, OR, USA).

Reverse transcription-quantitative polymerase chain reaction (RT-qPCR). Total RNA was isolated from the serum and harvested cells using TRIzol reagent (Thermo Fisher Scientific, Inc.). Then, RT-qPCR was performed using the One Step SYBR $^{\circledR}$ PrimeScript ${ }^{\text {TM }}$ RT-PCR kit (Takara Biotechnology Co., Ltd., Dalian, China) on an ABI 7300 Real-Time PCR system (Applied Biosystems; Thermo Fisher Scientific, Inc.), according to the manufacturer's protocol. The thermocycling conditions were as follows: $95^{\circ} \mathrm{C}$ for $30 \mathrm{sec}$, followed by 40 cycles of $95^{\circ} \mathrm{C}$ for $5 \mathrm{sec}$ and $60^{\circ} \mathrm{C}$ for $30 \mathrm{sec}$. All primers were synthesized by Sangon Biotech Co., Ltd. (Shanghai, China). The sequences of the primers were as follows: Hes1 forward, CTCCCGGCATTCCAAGCTA and reverse, AGC GGGTCACCTCGTTCATG; Notch1 forward, CACCCA TGACCACTACCCAGTT and reverse, CCTCGGACCAAT CAGAGATGTT; vascular endothelial growth factor (VEGF) forward, CTTGCCTTGCTGCTCTACCT and reverse, CTG CATGGTGATGTTGGACT; GAPDH forward, GAAGGT GAAGGTCGGAGTC and reverse, GAAGATGGTGATGGG ATTTC. The relative expression of Hes1, Notch1 and VEGF in each sample was normalized to the level of GAPDH using the $2^{-\Delta \Delta \mathrm{Cq}}$ method (19). The relative expression of miR-210 was examined using the Hairpin-it ${ }^{\mathrm{TM}}$ miRNAs qPCR Quantitation kit (GenePharma Co., Ltd.) according to the manufacturer's protocol, and U6 (RNU6B; GenePharma Co., Ltd.) was used for normalization.

Western blot analysis. Harvested cells were lysed in RIPA buffer (Beyotime Institute of Biotechnology, Shanghai, China), and the protein concentration was determined using a BCA Protein Assay kit (Beyotime Institute of Biotechnology). Proteins (30 $\mu 1 /$ lane) were separated by $8 \%$ SDS-PAGE. Following electrophoresis, the proteins on the gel were transferred to polyvinylidene difluoride membranes. The membranes were blocked with $5 \%$ non-fat milk at room temperature for $2 \mathrm{~h}$, and incubated with primary antibodies anti-VEGF (cat. no. ab46154; 1:1,000), anti-Notch1 (cat. no. ab52627; 1:1,000), anti-Hes1 (cat. no. ab71559; 1:1,000) and anti-GAPDH (cat. no. ab9485; 1:2,000) all purchased from Abcam (Cambridge, MA, USA) overnight at $4^{\circ} \mathrm{C}$. The next day, the membranes were washed and incubated with horseradish 
Table I. Clinical information of patients with ACI and healthy volunteers.

\begin{tabular}{lccr}
\hline Characteristic & Patients with ACI (n=40) & Healthy controls (n=40) & P-value \\
\hline Age, years (mean \pm SD) & $62.9 \pm 9.9$ & $63.4 \pm 10.8$ & 0.830 \\
Sex, male/female & $22 / 18$ & $21 / 19$ & 0.912 \\
Hypertension (\%) & $19(47.5 \%)$ & $13(32.5 \%)$ & 0.254 \\
Diabetes (\%) & $10(25.0 \%)$ & $5(12.5 \%)$ & 0.252 \\
Smoking (\%) & $19(7.45 \%)$ & $10(27.5 \%)$ & $0.037^{\text {a }}$ \\
Alcohol consumption history (\%) & $15(37.5 \%)$ & $10(25.5 \%)$ & 0.335
\end{tabular}

${ }^{\mathrm{a}} \mathrm{P}<0.05$ vs. healthy controls. ACI, acute cerebral infarction; SD, standard deviation.

A

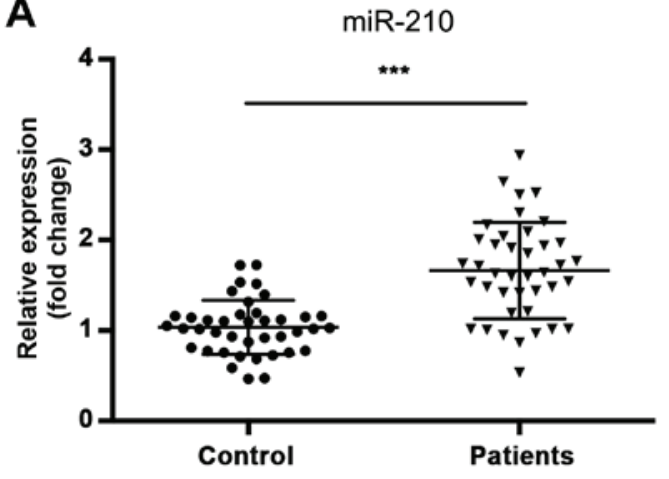

B

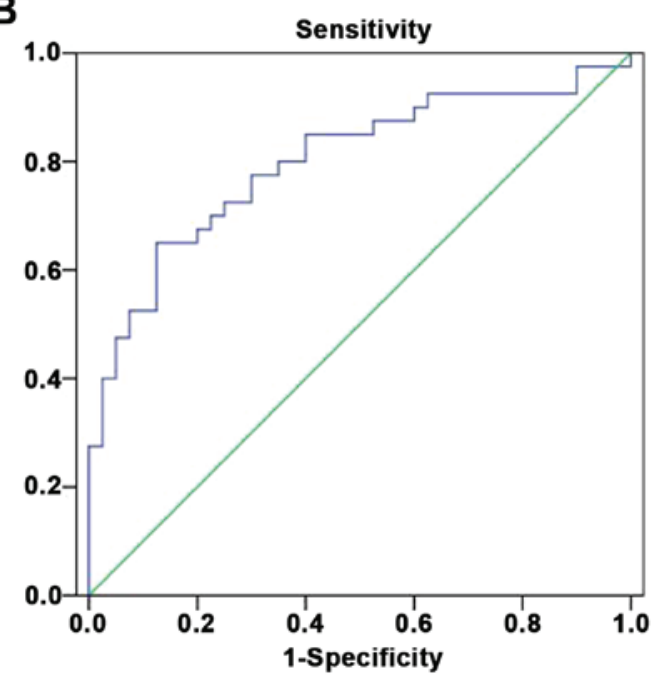

Figure 1. Expression of miR-210 in ACI. (A) Relative expression of miR-210 in the serum of patients with ACI and healthy controls; (B) Receiver operating characteristic curve analysis of miR-210. ${ }^{* * *} \mathrm{P}<0.001 \mathrm{vs}$. healthy controls. miR, microRNA; ACI, acute cerebral infarction.

peroxidase-conjugated secondary antibody (cat. no. A0208; 1:1,000; Beyotime Institute of Biotechnology) at room temperature for $45 \mathrm{~min}$. Finally, the membranes were incubated with enhanced chemiluminescence reagent (Beyotime Institute of Biotechnology) and imaged using the ChemiDoc ${ }^{\mathrm{TM}} \mathrm{XRS}+$ imaging system (Bio-Rad Laboratories, Inc., Hercules, CA, USA). The densitometry analysis was performed using Image Lab analysis software (version 2.1; Bio-Rad Laboratories, Inc., Hercules, CA, USA).
miR-210

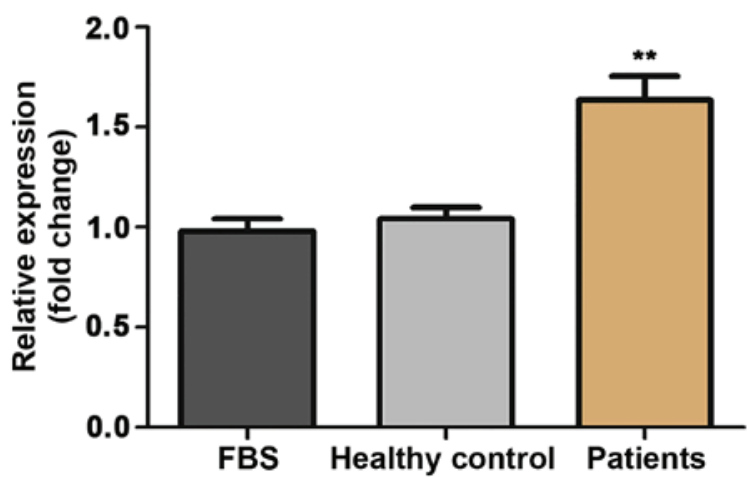

Figure 2. Expression of miR-210 in human umbilical vein endothelial cells treated with different mediums. ${ }^{* *} \mathrm{P}<0.01$ vs. FBS. FBS, fetal bovine serum; miR, microRNA.

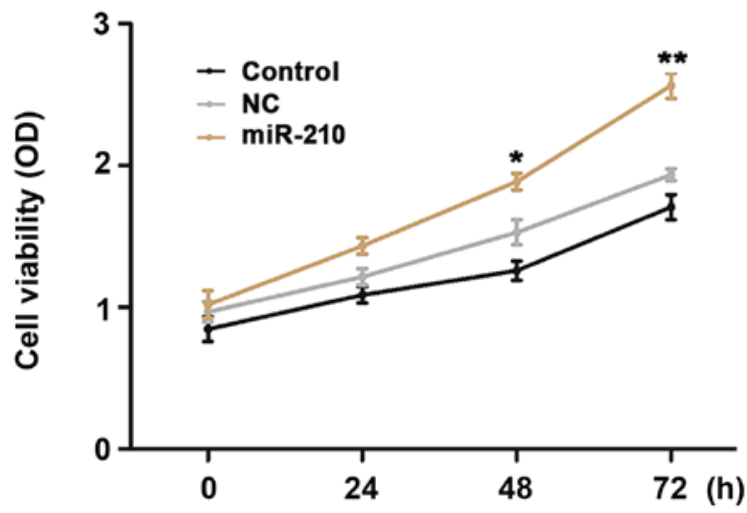

Figure 3. Proliferation rate of cells in different groups. ${ }^{*} \mathrm{P}<0.05,{ }^{* *} \mathrm{P}<0.01$ vs. control. Control, untransfected cells; NC, cells transfected with negative control miR-210 mimics; miR-210, cells transfected with miR-210 mimics; miR, microRNA.

Statistical analysis. Statistical analysis was performed using SPSS 19.0 software (IBM Corp., Armonk, NY, USA). Data are presented as the mean \pm standard deviation. Two independent sample t-test was performed to compare two groups, and one-way analysis of variance followed by Dunnett's post-hoc test was performed to compare multiple groups. A receiver operating characteristic (ROC) curve was used to evaluate the diagnostic performance of serum 
A

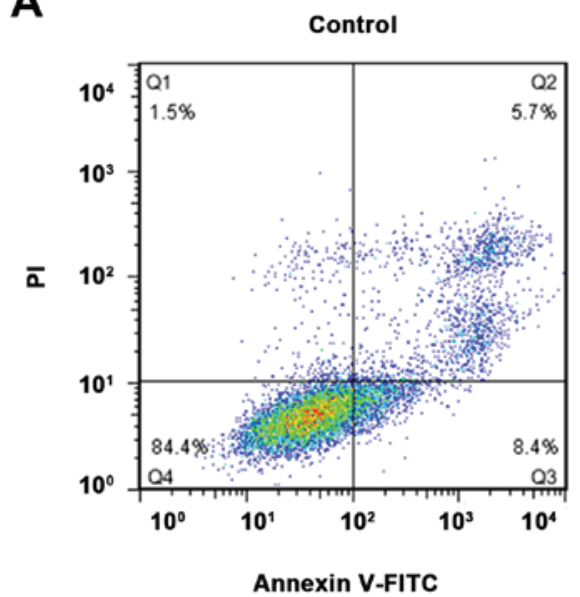

C

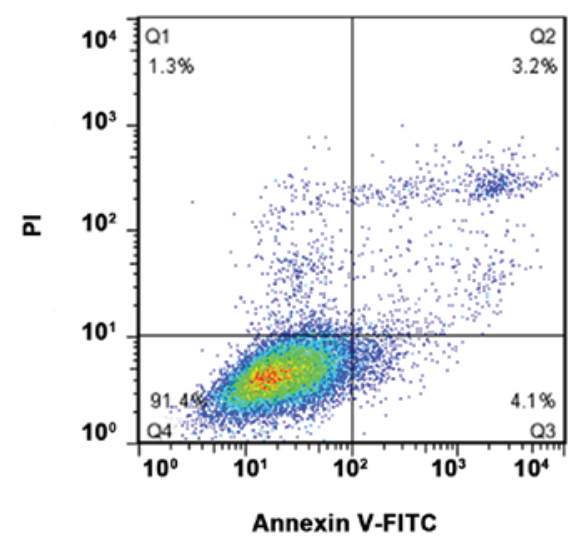

B

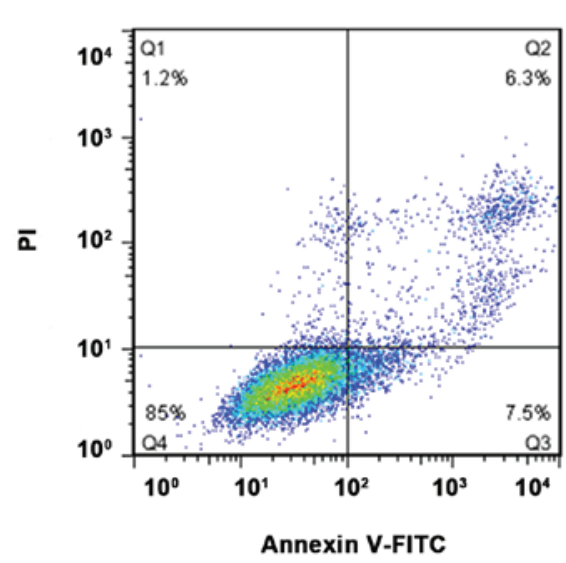

D

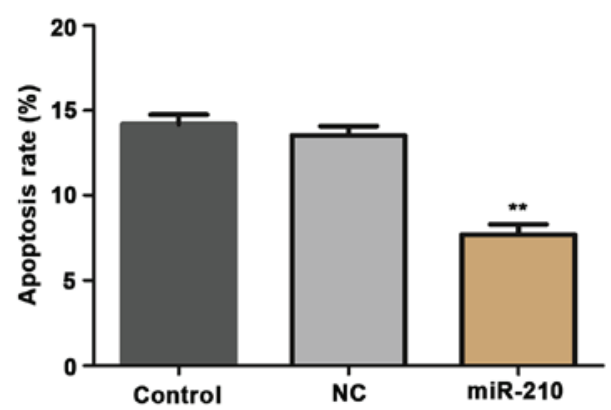

Figure 4. Apoptosis of cells in different groups: (A) Control, (B) NC and (C) miR-210. (D) Apoptosis rate was determined in each group. ${ }^{* *}$ P $<0.01$ vs. control. Control, untransfected cells; NC, cells transfected with negative control miR-210 mimics; miR-210, cells transfected with miR-210 mimics; miR, microRNA.

miR-210. $\mathrm{P}<0.05$ was considered to indicate a statistically significant difference.

\section{Results}

Expression of miR-210 is increased in the serum of patients with ACI. In order to investigate the potential role of miR-210 as a diagnostic marker, the level of miR-210 was compared in the serum of patients with ACI and healthy controls using RT-qPCR. The expression of miR-210 was significantly increased in the serum of patients with ACI compared with the healthy controls (Fig. 1A; $\mathrm{P}<0.001$ ). Furthermore, ROC curve analysis was performed to determine whether the serum level of miR-210 could distinguish ACI patients from healthy volunteers (Fig. 1B). The area under the curve (AUC) of miR-210 was 0.799 [95\% confidence interval (CI), 0.700-0.899], the optimum cut-off point was 1.397 , and the sensitivity and specificity at the cut-off point were 62.5 and $87.5 \%$, respectively.

Treatment of HUVECs with the serum from patients with ACI induces a significant increase in the expression of miR-210. To further explore the function of miR-210 in the pathogenesis of ACI, HUVECs were treated with the serum from patients with ACI or healthy controls, and the expression of miR-210 in HUVECs was examined using RT-qPCR. As shown in
Fig. 2, the expression of miR-210 was significantly increased in HUVECs treated with serum from patients with ACI compared with the healthy controls $(\mathrm{P}<0.01)$.

Upregulation of miR-210 affects the proliferation and apoptosis of HUVECs in vitro. HUVECs were transfected with miR-210 mimics in order to investigate the effects of miR-210 on the proliferation and apoptosis rates of cells using CCK-8 and flow cytometry methods. Compared with the untransfected cells, transient overexpression of miR-210 induced a significant increase in proliferation (Fig. 3; $\mathrm{P}<0.05$ at $48 \mathrm{~h}$, $\mathrm{P}<0.01$ at $72 \mathrm{~h})$ and a significant decrease in apoptosis rate (Fig. 4; $\mathrm{P}<0.01$ ) of HUVECs in vitro.

Upregulation of miR-210 affects the expression of Hesl, Notchl and VEGF in HUVECs in vitro. Finally, experiments were conducted to evaluate the underlying mechanism of the effect of miR-210 on proliferation and apoptosis of HUVECs. The expression of signaling proteins Hes1, Notch1 and VEGF in HUVECs was examined using RT-qPCR and western blotting methods. Transient overexpression of miR-210 induced a significant increase in the expression of Hes1 and VEGF in HUVECs at the mRNA $(\mathrm{P}<0.01$; Fig. 5$)$ and the expression of Hes1, Notch1 and VEGF in HUVECs at the protein $(\mathrm{P}<0.01$; Fig. 6) level. 

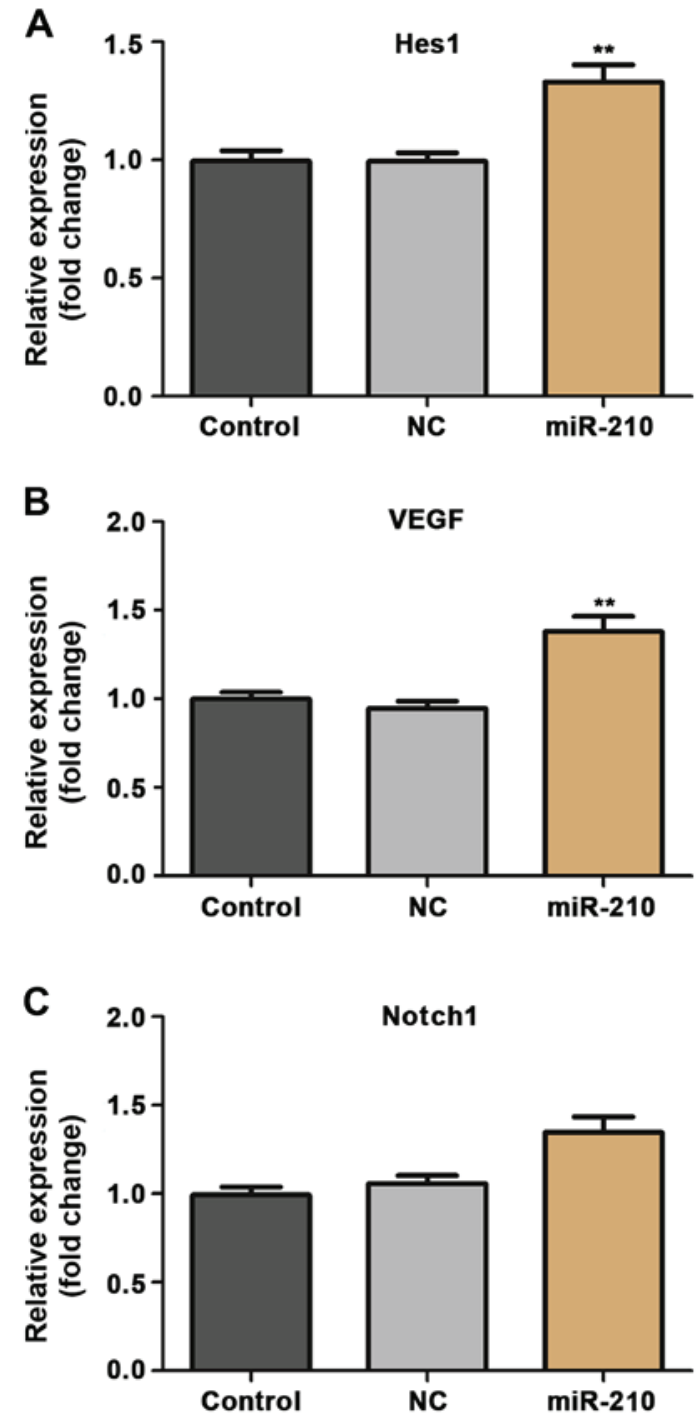

Figure 5. Relative mRNA expression of (A) Hes1, (B) Notch1 and (C) VEGF in human umbilical vein endothelial cells in different groups. ${ }^{* *} \mathrm{P}<0.01$ vs. control. Control, untransfected cells; $\mathrm{NC}$, cells transfected with negative control miR-210 mimics; miR-210, cells transfected with miR-210 mimics; miR, microRNA; VEGF, vascular endothelial growth factor.

\section{Discussion}

If no efficient medical treatment is administered within the first few $\mathrm{h}, \mathrm{ACI}$ can lead to poor prognosis and increased mortality rate among patients (20). Thus, effective early diagnostic biomarkers will help physicians to make rapid clinical decisions and improve clinical outcomes. The functions of circulating miRs as diagnostic markers of ACI have been discussed in numerous previous studies. Yuan et al (21) examined the plasma level of miR-26b, and its target calmodulin (CaM), in patients with ACI, and identified that miR-26b was upregulated and $\mathrm{CaM}$ was downregulated in the plasma of patients with ACI compared with healthy controls. The same study also demonstrated that elevated CaM and decreased miR-26b expression in the plasma of patients was associated with poor clinical outcomes. Zhou and Zhang (17) demonstrated that the plasma levels of miR-21 and miR-24 may act as diagnostic markers during the early stage of ACI. Weng et al (22) identified that the plasma concentration of miR-124 is a promising
A
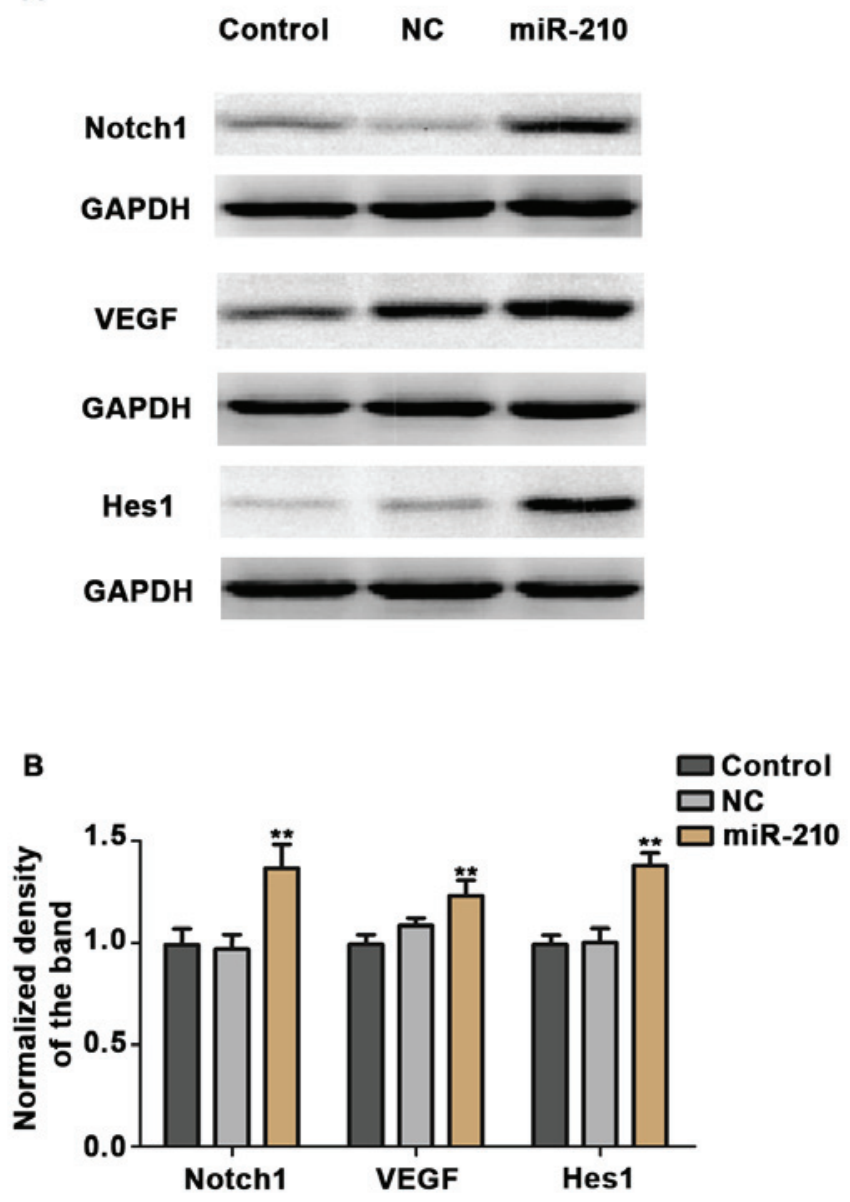

Figure 6. (A) Protein expression of Hes1, Notch1 and VEGF in human umbilical vein endothelial cells in different groups. (B) Relative protein expression levels were semi-quantified. ${ }^{* *} \mathrm{P}<0.01$ vs. control. Control, untransfected cells; $\mathrm{NC}$, cells transfected with negative control miR-210 mimics; miR-210, cells transfected with miR-210 mimics; miR, microRNA; VEGF, vascular endothelial growth factor.

candidate biomarker for early detection of cerebral infarction. In the present study, it was observed that the expression of miR-210 was significantly increased in the serum of patients with ACI compared with normal controls. Furthermore, ROC curve analysis indicated that the AUC of miR-210 was 0.799 (95\% CI, 0.700-0.899), the optimum cut-off point was 1.397 , and the sensitivity and specificity at the cut-off point were 62.5 and $87.5 \%$, respectively. These results indicate that the serum level of miR-210 is an efficient biomarker that can distinguish patients with ACI from healthy individuals. In summary, these data suggest that circulating miR-210 has potential as a novel biomarker for the early diagnosis of ACI.

Hypoxia and the production of excessive reactive oxygen species are frequently observed in the brain tissue of patients with cerebral ischemia $(23,24)$, and these extreme conditions can lead to apoptosis of cells (including neurons and vascular endothelial cells) in the brain. It has been reported that the aberrant apoptosis of vascular endothelial cells in cerebral vessels can aggravate secondary brain injury after cerebral infarction (25). Thus, proliferation and angiogenesis of endothelial cells are key events for recovery of the brain after cerebral infarction. Previous studies have suggested that 
miRs can regulate the proliferation and migration of endothelial cells after cerebral infarction. Zhang et al (26) reported that miR-433 was downregulated in hypoxia conditions, and miR-433 could regulate the proliferation and migration of HUVECs. Chen et al (27) demonstrated that miR-145 could increase proliferation and migration of endothelial progenitor cells in a cerebral infarction mice model through regulating the c-Jun N-terminal kinase signal pathway. Yuan et al (28) reported that the polymorphism of MMP-9 at the miR-491-5p binding site was associated with the risk of cerebral infarction in a Chinese population. Lou et al (29) demonstrated that miR-210 could activate the Notch signaling pathway and participate in the process of angiogenesis following cerebral ischemia.

In the present study, the effect of miR-210 on the proliferation and apoptosis of HUVECs was evaluated. HUVECs were treated with serum from patients with ACI or healthy controls, and it was identified that treatment of HUVECs with serum from patients with ACI induced a significant increase in the expression of miR-210 compared with the controls. This suggested that the expression of miR-210 was significantly upregulated in HUVECs under ACI conditions. Furthermore, transient overexpression of miR-210 induced a significant increase in cell proliferation and a significant decrease in cell apoptosis. It also induced an increase in the expression of VEGF, Notch1 and Hes1 at the mRNA and protein levels. These results indicated that miR-210 may serve a protective function in ACI through facilitating the proliferation of vascular endothelial cells and angiogenesis.

In conclusion, the present study demonstrates that miR-210 is upregulated in the serum of patients with ACI, and miR-210 may be involved in the pathogenesis of ACI through regulating the proliferation and apoptosis of endothelial cells. This suggests that miR-210 has potential as a diagnostic tool and therapeutic target for the early diagnosis and management of ACI.

\section{References}

1. Roth GA, Johnson CO, Nguyen G, Naghavi M, Feigin VL, Murray CJ, Forouzanfar MH and Vos T: Methods for estimating the global burden of cerebrovascular diseases. Neuroepidemiology 45 : 146-151, 2015.

2. Wu X, Zhu B, Fu L, Wang H, Zhou B, Zou S and Shi J: Prevalence, incidence and mortality of stroke in the chinese island populations: A systematic review. PLoS One 8: e78629, 2013.

3. Ji JF and Ma XH: Effect of baculovirus P35 protein on apoptosis in brain tissue of rats with acute cerebral infarction. Genet Mol Res 14: 9353-9360, 2015.

4. Saver JL, Goyal M, Bonafe A, Diener HC, Levy EI, Pereira VM, Albers GW, Cognard C, Cohen DJ, Hacke W, et al: Stent-retriever thrombectomy after intravenous t-PA vs. t-PA alone in stroke. N Engl J Med 372: 2285-2295, 2015.

5. Haupt WF, Chopan G, Sobesky J, Liu WC and Dohmen C: Prognostic value of somatosensory evoked potentials, neuron-specific enolase and S100 for short-term outcome in ischemic stroke. J Neurophysiol 115: 1273-1278, 2016.

6. Li K, Jia J, Wang Z and Zhang S: Elevated serum levels of NSE and S-100beta correlate with increased risk of acute cerebral infarction in asian populations. Med Sci Monit 21: 1879-1888, 2015.

7. Xiao D, Liu H, Zhang $\mathrm{H}$ and Luo Y: Impact of cystatin $\mathrm{C}$ levels on infarct size and hemorrhage volume in acute cerebral stroke. J Neurol 259: 2053-2059, 2012.

8. Lee RC, Feinbaum RL and Ambros V: The C. Elegans heterochronic gene lin-4 encodes small RNAs with antisense complementarity to lin-14. Cell 75: 843-854, 1993.
9. Backes C, Meese E and Keller A: Specific miRNA disease biomarkers in blood, serum and plasma: Challenges and prospects. Mol Diagn Ther 20: 509-518, 2016.

10. Sun D, Li A, Feng H and Wang M: NTSMDA: Prediction of miRNA-disease associations by integrating network topological similarity. Mol Biosyst 12: 2224-2232, 2016.

11. Zhu K, Liu D, Lai H, Li J and Wang C: Developing miRNA therapeutics for cardiac repair in ischemic heart disease. J Thorac Dis 8: E918-E927, 2016.

12. Mehta R, Otgonsuren M, Younoszai Z, Allawi H, Raybuck B and Younossi Z: Circulating miRNA in patients with non-alcoholic fatty liver disease and coronary artery disease. BMJ Open Gastroenterol 3: e000096, 2016.

13. Zhang JY, Gong YL, Li CJ, Qi Q, Zhang QM and Yu DM: Circulating MiRNA biomarkers serve as a fingerprint for diabetic atherosclerosis. Am J Transl Res 8: 2650-2658, 2016.

14. Zhao C, Lu F, Chen H, Zhao F, Zhu Z, Zhao X and Chen H: Clinical significance of circulating miRNA detection in lung cancer. Med Oncol 33: 41, 2016.

15. Chen Y, Song Y, Huang J, Qu M, Zhang Y, Geng J, Zhang Z, Liu J and Yang GY: Increased circulating exosomal miRNA-223 is associated with acute ischemic stroke. Front Neurol 8: 57, 2017.

16. Sørensen SS, Nygaard AB, Nielsen MY, Jensen $K$ and Christensen T: miRNA expression profiles in cerebrospinal fluid and blood of patients with acute ischemic stroke. Transl Stroke Res 5: 711-718, 2014.

17. Zhou $\mathrm{J}$ and Zhang J: Identification of miRNA-21 and miRNA-24 in plasma as potential early stage markers of acute cerebral infarction. Mol Med Rep 10: 971-976, 2014

18. Sepramaniam S, Tan JR, Tan KS, DeSilva DA, Tavintharan S, Woon FP, Wang CW, Yong FL, Karolina DS, Kaur P, et al: Circulating microRNAs as biomarkers of acute stroke. Int J Mol Sci 15: 1418-1432, 2014.

19. Rao X, Huang X, Zhou Z and Lin X: An improvement of the $2^{\wedge}$ (-delta delta CT) method for quantitative real-time polymerase chain reaction data analysis. Biostat Bioinforma Biomath 3: 71-85, 2013.

20. Arboix A, Garcia-Eroles L, Oliveres M, Comes E, Sánchez MJ and Massons J: Malignant middle cerebral artery infarction: A clinical study of 32 patients. Rev Invest Clin 67: 64-70, 2015.

21. Yuan M, Tang Y, Zhou C, Liu F, Chen L and Yuan H: Elevated plasma CaM expression in patients with acute cerebral infarction predicts poor outcomes and is inversely associated with miR-26b expression. Int J Neurosci 126: 408-414, 2016.

22. Weng H, Shen C, Hirokawa G, Ji X, Takahashi R, Shimada K, Kishimoto $\mathrm{C}$ and Iwai N: Plasma miR-124 as a biomarker for cerebral infarction. Biomed Res 32: 135-141, 2011.

23. Page S, Munsell A and Al-Ahmad AJ: Cerebral hypoxia/ischemia selectively disrupts tight junctions complexes in stem cell-derived human brain microvascular endothelial cells. Fluids Barriers CNS 13: 16, 2016.

24. Yang Z, Zhong L, Zhong S, Xian R and Yuan B: Hypoxia induces microglia autophagy and neural inflammation injury in focal cerebral ischemia model. Exp Mol Pathol 98: 219-224, 2015.

25. Shen J, Zhu Y, Huang K, Jiang H, Shi C, Xiong X, Zhan R and Pan J: Buyang huanwu decoction attenuates H2O2-induced apoptosis by inhibiting reactive oxygen species-mediated mitochondrial dysfunction pathway in human umbilical vein endothelial cells. BMC Complement Altern Med 16: 154, 2016.

26. Zhang L, Zhang Y, Zhang X, Zhang Y, Jiang Y, Xiao X, Tan J, Yuan W and Liu Y: MicroRNA-433 inhibits the proliferation and migration of HUVECs and neurons by targeting hypoxia-inducible factor 1 alpha. J Mol Neurosci 61: 135-143, 2017.

27. Chen R, Chen S, Liao J, Chen X and Xu X: MiR-145 facilitates proliferation and migration of endothelial progenitor cells and recanalization of arterial thrombosis in cerebral infarction mice via JNK signal pathway. Int J Clin Exp Pathol 8: 13770-13776, 2015.

28. Yuan M, Zhan Q, Duan X, Song B, Zeng S, Chen X, Yang Q and Xia J: A functional polymorphism at miR-491-5p binding site in the 3'-UTR of MMP-9 gene confers increased risk for atherosclerotic cerebral infarction in a Chinese population. Atherosclerosis 226: 447-452, 2013.

29. Lou YL, Guo F, Liu F, Gao FL, Zhang PQ, Niu X, Guo SC, Yin JH, Wang Y and Deng ZF: miR-210 activates notch signaling pathway in angiogenesis induced by cerebral ischemia. Mol Cell Biochem 370: 45-51,2012.

This work is licensed under a Creative Commons Attribution-NonCommercial-NoDerivatives 4.0 International (CC BY-NC-ND 4.0) License. 